\title{
Special Issues of AMR on the Occasion of the 85th Birthday of Harold M. Swartz
}

\author{
Ann Barry Flood ${ }^{1}$ (D) . Steven G. Swarts ${ }^{2}$ (D) $\cdot$ Bernard Gallez $^{3}$ (D) \\ Murali C. Krishna ${ }^{4}$
}

Published online: 17 September 2021

(c) The Author(s), under exclusive licence to Springer-Verlag GmbH Austria, part of Springer Nature 2021

\section{Introduction}

On the occasion of his 85th birthday, a group of colleagues mobilized to create a festschrift in honor of the principal scientific contributions to the field of magnetic resonance of Harold M. Swartz, M.D., Ph.D., M.S.P.H. (Hal Swartz). Stemming from his initial passion for medical science, a major overarching theme of his work is to identify how to use the special attributes of electron paramagnetic resonance (EPR) to create novel clinical applications to improve healthcare and medical science. Over the past approximately 60 years, Hal has made major contributions that have both increased the fundamental understanding of certain physiological and pathophysiological mechanisms and created novel ways to measure these processes in vivo using EPR.

In this first issue, the contributions in Hal's honor focus on in vivo oximetry, either by presenting comprehensive reviews or by advancing the field through new studies. In the second issue, the papers honor Hal's contributions to retrospective dosimetry, in the context of assessing the dose of individuals potentially exposed in a large-scale incident involving ionizing radiation. Articles in the second issue also pay tribute to Hal's scientific guidance in the area of clarifying the nature and clinical meaningfulness of EPR signals that are detected in cells and tissues when assessing their free radicals and paramagnetic ions. Finally, articles in the second issue pay homage to Hal's contributions to the EPR instrumental developments needed to perform in vivo measurements (including technical advancements in resonators, magnets, and

Ann Barry Flood

ann.barry.flood@dartmouth.edu

1 Department of Radiology, Geisel School of Medicine, Dartmouth College, Hanover, NH, USA

2 Department of Radiation Oncology, School of Medicine, University of Florida, Gainesville, FL, USA

3 Biomedical Magnetic Resonance, Louvain Drug Research Institute, Université Catholique du Louvain, Brussels, Belgium

4 Radiation Biology Branch, National Cancer Institute, Bethesda, MD, USA 
microwave bridges as well as software advances, human factors considerations, and the development of paramagnetic probes to use for in vivo measurements).

\section{Brief Biography of Harold Swartz}

Hal Swartz was born on June 22, 1935 in Chicago, IL (home of his beloved Cubbies!) to Sidney "Tim" Swartz and Amy Saks Swartz. Like his father before and daughter Sharon afterward, Hal attended the University of Chicago, where he learned to stretch his mind and his horizons; he also attended Loyola University where he got most of his premedical training. He graduated with an M.D. from the University of Illinois@Chicago, married his high school sweetheart, Bonnie Pashkow, and soon began raising his initial family (Sharon Swartz b1959 and Daniel Swartz b1962). The Army soon 'called back' his part of the bargain for their year of supporting his tuition and medical training, providing him with 'the opportunity' to serve as an Army medical officer. Hal eventually served in the Army almost 10 years, reaching the rank of lieutenant colonel.

The Army was very interested in his research in radiation biology and in due course supported his additional training in radiation biophysics (when he earned an MSPH from University of North Carolina@Chapel Hill) and in the effects of oxygen on cells (when he earned a Ph.D. in biochemistry/biophysics from Georgetown University). His first principal deployment while in the Army was at the Utah base, Dugway Proving Ground, where he served both as a general practitioner for the military and their families and for other civilians stationed there and as a field military medical officer during biological and chemical testing. This experience fueled Hal's interests in preventing the effects of biological, chemical and especially radiological warfare on military and civilians and led to his subsequent deployment to the Walter Reed Army Medical Center [where he started as the untrained head of a small magnetic resonance EPR/NMR facility and grew to be the Head of the Departments of Biophysics (1966-69) and Biological Chemistry (1970)]. Although the original uses of EPR spectroscopy were in physics, Hal was among the earliest who quickly saw its potential to inform other fields such as structural organic chemistry, biology and especially medicine.

After serving in the Army, he moved to a position as Professor at the Medical College of Wisconsin (MCW) and Director of Radiation Biology and Biophysics (1970-1980). In 1975, he started the first of three EPR Centers he founded, codirecting the National Biomedical ESR Center at MCW with James S. Hyde, Ph.D. At MCW, he began his passion to advance medical education as well as train M.D.Ph.D.'s to improve medical science and practiced as a radiologist, becoming board certified in nuclear medicine.

After 10 years at MCW, he moved to the University of Illinois@ Urbana-Champaign (UIUC) to become Associate Dean for Academic Affairs at the Medical School. In 1985, while at UIUC, he founded and co-directed with R. Linn Belford, Ph.D., a second EPR Center (The University of Illinois EPR Research Center), invigorated an MD$\mathrm{PhD}$ Program that grew to be arguably the largest such program in the world, concurrently training up to 150 scholars in $40+$ graduate programs. Hal also began a second 
marriage and new partnership (with Ann Barry Flood, Ph.D.) and acquired a new family of teenaged children (Derek Flood b1967 and Shannon Flood b1970). He then spent a sabbatical year at Johns Hopkins and an additional half year at Oxford University before joining the faculty at the Geisel School of Medicine at Dartmouth (Geisel) and founding and directing a third EPR Center (The EPR Center for the Study of Viable Systems), devoted to advancing the biological and clinical applications of EPR. At Geisel, he also founded and directed the Dartmouth Center for Medical Countermeasures Against Radiation (funded by NIAID to advance EPR dosimetry) and obtained his second Program Project Grant from NIH (funded by NCI for advancing EPR oximetry).

To date, he has more than 570 publications and 9 patents and remains fully active (but does state that he is planning on retiring in 2035 when he turns 100). While not highlighted in this festschrift, Hal has also published in areas of his other passions such as medical education and mentoring young researchers. For example, in addition to directing the previously mentioned large MD-PhD Program at UIUC, Hal directed the Program for Medical Humanities and Social Sciences at UIUC's Medical School; he founded and directed a PhD program in biophysics at MCW; he founded and directed the MS and PhD Programs at the Dartmouth Institute for Healthcare and Clinical Practice, and he reinvigorated the MD-PhD Program at Geisel.

Hal has won several prestigious awards for his outstanding scientific contributions, e.g., he received The International Zavoisky Award (2005); he was a founding board member of the Society for Magnetic Resonance in Medicine (SMRM) which eventually became the International Society for Magnetic Resonance in Medicine, to which he was appointed as a Fellow (1997) as well as of the International EPR Society (2005); he was awarded a Silver Medal in Biomedicine from the International EPR Society (1994) and a Silver Medal from the SMRM (1993); he was named the Alma Hass Milham Distinguished Chair in Clinical Medicine at Geisel School of Medicine at Dartmouth (2012). He is also widely recognized for his generous and exceptional professional services to the field of in vivo EPR as a founder of several societies or divisions within them, as a president or other type of officer, and as serving on the executive committee of numerous international societies in magnetic resonance [e.g., Hal founded the Gordon Conference on Magnetic Resonance in Biology (1974); he was awarded a Special Gold Medal (2005) as Founder of the International EPR Society and served as President and scientific host of meetings for it (1990-93) and for the International Society for Oxygen Transport to Tissue (1998 and 2015) and the International Association of Biological and EPR Radiation Dosimetry (2015)]. In 2007, he co-founded (with Ann Flood) a small company, Clin-EPR, LLC, with the mission to advance scientific studies in in vivo EPR. For more details see his full CV at https://www.clin-epr.com/team. 


\section{Connecting Hal's Major Scientific Contributions to the Articles in this Festschrift}

There are arguably three main threads to Hal's lifetime of scientific contributions to the field of magnetic resonance, which we emphasize in this collection. Issue 1 emphasizes the first thread: Hal's major contributions related to measuring and understanding the biological effects of oxygen. An early and persistent theme to his work is to contribute to the understanding of how and why oxygen has both a lifegiving, metabolic function and a toxic effect on biological systems, i.e., to understand both the physiology and the pathophysiology associated with oxygen levels. His approach, based fundamentally on magnetic resonance, combines both of his own major interests in biophysics and in medicine. Beginning with his Ph.D. thesis work, he established the occurrence of an oxygen effect during freezing damage to cells. He has used EPR in particular to look for evidence of gradients within a cell and its immediate microenvironment and how and why intracellular oxygen gradients are related to pathophysiology. While instrumental developments associated with EPR will be featured in Issue 2, such developments have gone hand-in-hand with the evolution of the knowledge about the effects of oxygen on biological systems and the need to measure them to realize Hal's other passion and major contribution: developing clinical applications of EPR to improve the diagnosis, prognosis, and treatment of human disease. For a more comprehensive review of his work involving oxygen and oximetry, see Harold Swartz' "History of EPR oximetry by Swartz Group" in the second issue.

\section{Issue 1: Measuring and Understanding the Biological Effects of Oxygen}

The 13 articles in this issue contribute to these themes related to oxygen and EPR oximetry by raising fundamental questions, reviewing the current state of the science and gaining insights from such an overview, and providing new evidence about the effect of oxygen and the possibilities for improving clinical care.

The first three articles discuss theoretical approaches in using EPR to study oxygen. For example, the first article refers to the well-established principle that measuring oxygen using EPR depends on the electron spin relaxation time of oxygen being short, relative to the relaxation time of the sensor molecules. However, the likely effects on the EPR spectra from the surrounding environment have not to date been comprehensively evaluated. Sandra and Gareth Eaton, in their review, "EPR spectra and electron spin relaxation of $\mathrm{O}_{2}$," rectify this problem. They present a comprehensive overview of the literature to compare the electron spin relaxation of oxygen when it is in a gas phase, dissolved in fluid solutions and in frozen solutions. Karol Subczinski and his colleagues in their review, "Factors determining barrier properties to oxygen transport across model and cell plasma membranes based on EPR spin-label oximetry", focus on the many factors that can decrease oxygen permeation across cell plasma membranes. Of special 
interest is whether the plasma membrane could ever form a barrier to the free diffusion of oxygen into the cell interior and thus to the cell's DNA. This is of special interest to them because of its importance to understanding the role of oxygen in FLASH radiotherapy. Gary Angles, Rachel Dotson, and Sally Pias, in their original work using simulations, "Atomistic simulations modify interpretation of spin-label oximetry data. Part 1: Intensified water-lipid interfacial resistances," are also interested in modeling and understanding oxygen diffusion across membranes. They are particularly interested in the effects of membrane cholesterol, which is thought to slow oxygen diffusion and reduce oxygen permeability of the membrane. Using all-atom molecular dynamics simulation data and an updated model of oxygen, they seek to determine the location of maximum resistance to permeation. They found evidence of a spike not detected by EPR but which had been predicted in early theoretical work; this discrepancy underscores the potential value of using atomic resolution models to understand how to interpret probebased measurements.

The next three articles are about developing and evaluating techniques to assess oxygen using EPR. Murali Krishna and Subu Subramanian, in their review, "The development of time-domain in vivo EPR imaging at NCI," discuss the history and technical developments, focusing on efforts in their laboratory at the NCI that kindled the current attempts to develop FT-EPR imaging. They are now able to routinely generate fast in vivo EPR images of small animals using time-domain EPR. Although FT-EPR imaging requires administration of a non-toxic biocompatible paramagnetic probe in the tissue of interest, EPR imaging holds a unique promise of quantitatively mapping the in vivo tissue oxygen distribution non-invasively. This is particularly important in cancer biology due to the hypoxic nature of many tumors and their subsequent resistance to radiotherapy and other treatments. Stemming from similar interests, Boris Epel and Howard Halpern, in "EPR oxygen imaging workflow with MATLAB image registration toolbox," describe their laboratory's development of an animal bed with registration guides and a MATLAB toolbox that allows for registering EPR images and analyzing the data. Eunice Chen and her colleagues from the EPR Center at Dartmouth, focusing on EPR spectroscopy rather than EPR imaging, present an in vivo study of oxygen measurements made in normal tissue in the brain and hindleg muscle of rabbits and a post-study biological evaluation of the tissues surrounding the resonator site and a control site in the same rabbit. Their study, "Evaluation of a refined implantable resonator for deep-tissue EPR oximetry in the clinic", presents initial evidence of the feasibility and safety of an implantable resonator that is potentially capable of repeatedly measuring tumors at any depth. The latter is a significant improvement over EPR oxygen sensors that are limited by their sensitive depth.

The next two articles present reviews of the physiology and pathophysiology of oxygen, in each case focused particularly on the brain. Mada Hashem and Jeff Dunn, in "Brain oximetry and the quest for quantified metabolic rate: Applications using MRI and Near-Infrared Spectroscopy," compare and contrast MRI-based and NIRSbased methods to assess brain health. Where available, they review evidence from animal models, neonates and adults under normal and different physiological conditions. They propose combining techniques into a multimodel approach that provides 
better information about the oxidative metabolism in the brain. John Weaver and Jim Liu, in recognition of the seminal work by Hal Swartz' group (across a time period that crosses several of his EPR Centers), focus initially on recounting this pioneering work in low frequency technology, which overcame the difficulties of performing in vivo EPR spectroscopy and allowed obtaining scientific evidence unique to EPR techniques. In the second half of "A review of low-frequency EPR technology for the measurement of brain $\mathrm{pO}_{2}$ and oxidative stress," John and Jim focus on studies of partial $\mathrm{pO}_{2}$ changes and oxidative stress under various physiological and pathophysiological conditions.

The next two articles present particularly valuable, up-to-date comprehensive reviews of EPR and other methods for measuring oxygen in tissues (not including in blood). Bernard Gallez, in "Oxygenation status in normal tissues, pathologi-

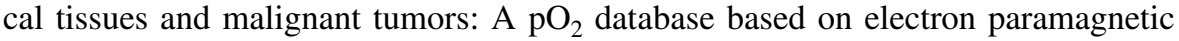
resonance (EPR) oximetry measurements", begins by defining the methods that are included in the term 'EPR oximetry' and reviewing how these techniques assess oxygen status in normal and pathological tissues and under challenges such as during oxygen enriched breathing or after pharmacological interventions. Overall, this paper provides a large comprehensive database on $\mathrm{pO}_{2}$ values that have been recorded in different tissues and under different physiological conditions. Peter Vaupel, Ann Flood and Hal Swartz focus on the explanations for why tumors are typically hypoxic and the clinical implications, both for prognosis and for understanding and potentially overcoming hypoxia-related tumor resistance to treatments. In "Oxygenation status of malignant tumors vs. normal tissues: Critical evaluation and updated data source based on direct measurements with $\mathrm{pO}_{2}$ microsensors", they also present a comprehensive and updated review of the data, based on measurements made using polarographic $\mathrm{pO}_{2}$ microsensors, and discuss the desirable characteristics of clinical methods to assess tumor oxygen.

The final three articles in Issue 1 of the festschrift for Hal Swartz are a fitting conclusion to this section on oximetry, because they each describe novel clinical applications of techniques to assess oxygen. In Eunice Chen, Sassan Hodge and Ben Williams' study, "Tissue oxygen profiling using EPR oximetry to improve wound healing after radiation," they used a rodent model for wound healing. EPR oximetry was used to profile oxygen before and after treatment by radiation, surgery and hyperbaric oxygen therapy. They found significant evidence that irradiation compromised wound healing, and that wound healing was poorer in irradiated tissues that had lower oxygenation early on. They conclude that oxygen profiling with EPR could be used to identify when to use oxygen therapy to improve wound healing. Paul Buehler, Ann Flood, and Hal Swartz present a different clinical application of EPR oximetry: evaluating blood transfusion products by measuring how well they actually deliver oxygen to the targeted tissue. In "Expanding EPR oximetry into transfusion medicine" the authors point out that the effectiveness of transfusions depends on the recipient's physiological tolerance to acute anemia as well as the quality of the donor units (e.g., age in storage). For these reasons, the indirect 
methods for assessing the effectiveness of transfusions that are currently used, while not useless, also do not directly assess whether or how well tissues are in fact oxygenated-which EPR oximetry is uniquely capable of assessing. They conclude that EPR oximetry could be used both to assess the effectiveness of different types of blood products and also to evaluate the effectiveness of transfusions for individuals. Finally, Brian Pogue and colleagues in their article, "Review of tissue oxygenation sensing during radiotherapy based upon Cherenkov-excited luminescence imaging," discuss using the unique capability of Cherenkov light excitation to directly assess $\mathrm{pO}_{2}$ in the tumor during radiation therapy.

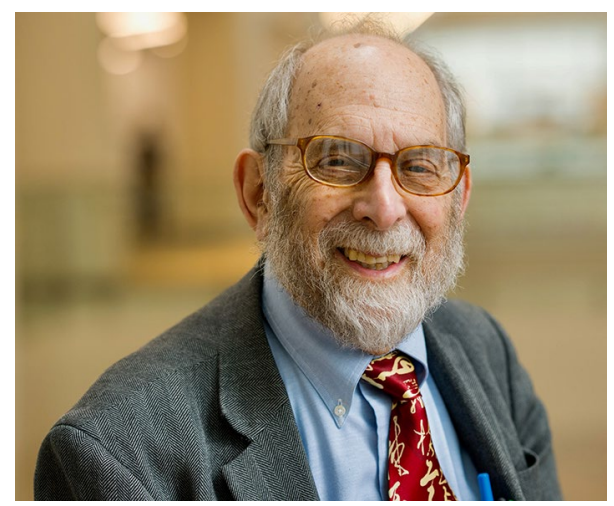

Acknowledgements Ann served as the lead editor for this festschrift. Steve served as the lead editor for all articles on which she or Hal were coauthors and was the topic editor for dosimetry related articles. Bernard served as topic editor for oximetry related articles, and Murali was the topic editor for free radicals and EPR instrumental developments.

Publisher's Note Springer Nature remains neutral with regard to jurisdictional claims in published maps and institutional affiliations. 